\title{
Atendimento Odontológico a paciente em uso de Bifosfonatos: Relato de caso
}

\author{
Dental care to patients using Biposphonates: Case report \\ Atención Dental al paciente con Biposfonatos: Reporte de caso
}

Recebido: 10/07/2021 | Revisado: 14/07/2021 | Aceito: 15/07/2021 | Publicado: 24/07/2021

\author{
Elizama de Lima Amazonas \\ ORCID: https://orcid.org/0000-0003-3337-0721 \\ Universidade Paulista, Brasil \\ lilica1506@gmail.com \\ Darcleia Carneiro Sacht \\ ORCID: https://orcid.org/0000-0003-0014-1682 \\ Universidade Paulista, Brasil \\ darcleiasouza@live.com \\ Ranniely de Souza Alves \\ ORCID: https://orcid.org/0000-0003-1792-4085 \\ Universidade Paulista, Brasil \\ rannielysouza9@gmail.com \\ Nely Cristina Medeiros Caires \\ ORCID: https://orcid.org/0000-0003-3094-4064 \\ Universidade Paulista, Brasil \\ nelycaires@gmail.com
}

\begin{abstract}
Resumo
A osteoporose é uma doença sistêmica progressiva do esqueleto, caracterizada pela redução da densidade óssea e deterioração microestrutura do tecido ósseo. Para o tratamento, tem sido preconizado como terapia antirreabsortiva de $1^{\mathrm{a}}$ escolha, o uso de Bifosfonatos (BFs). O objetivo deste trabalho é descrever por meio de relato de caso, protocolo de atendimento odontológico a paciente portador de Osteoporose e em uso de BFFs. O trabalho foi submetido e aprovado pelo Comitê de Ética em Pesquisa (CEP), parecer $n^{\circ}$ : 3.646.508/2019. Paciente gênero feminino, 30 anos, melanoderma, portadora de Osteoporose, foi encaminhada para realização de tratamento odontológico. No momento da Anamnese relatou fazer uso de Alendronato de Sódio 70mg/dia. Após o exame clínico, exames laboratoriais para dosagem de CTX-I e avaliação radiográfica, apresentou-se o plano de tratamento sugerido: cirurgia de aumento de coroa clínica e posterior tratamento endodôntico e reabilitação oral do dente 25 , que foi prontamente aprovado pela paciente e assinado o Termo de Consentimento Livre e Esclarecido (TCLE). Uma semana antes da realização do procedimento cirúrgico de aumento de coroa clínica, foi solicitado a suspensão do Alendronato de sódio. Após o tratamento endodôntico, foi feita a reabilitação protética, restituindo a função mastigatória e estética dos dentes, devolvendo a autoestima à paciente. Concluiu-se no presente estudo que é possível realizar o tratamento odontológico do paciente em terapia com Bifosfonatos com segurança, desde que realizada anamnese bem conduzida e detalhada e adotados protocolos seguros de tratamento, sempre visando a qualidade de vida e satisfação do paciente.
\end{abstract}

Palavras-chave: Osteoporose; Bifosfonatos; Osteonecrose; Odontólogos.

\begin{abstract}
Osteoporosis is a progressive systemic disease of the skeleton, characterized by reduced bone density and microstructure deterioration of bone tissue. For treatment, the use of bisphosphonates (BFFs) has been recommended as the first choice anti-resorptive therapy. The objective of this work is to describe, through a case report, a dental care protocol for a patient with Osteoporosis and using BFs. The work was submitted and approved by the Research Ethics Committee (CEP), opinion No.: 3.646.508/2019. Female patient, 30 years old, black, with osteoporosis, was referred for dental treatment. At the time of the Anamnesis, he reported using Alendronate Sodium 70mg/day. After clinical examination, laboratory tests for CTX-I dosage and radiographic evaluation, the suggested treatment plan was presented: clinical crown augmentation surgery and subsequent endodontic treatment and oral rehabilitation of tooth 25, which was promptly approved by the patient and signed the Informed Consent Term (TCLE). One week before the surgical procedure for clinical crown augmentation, the suspension of Alendronate sodium was requested. After the endodontic treatment, the prosthetic rehabilitation was carried out, restoring the masticatory and aesthetic function of the teeth, restoring the patient's self-esteem. It was concluded in the present study that it is possible to carry out the dental treatment of patients in bisphosphonate therapy safely, if a well-conducted and detailed anamnesis is carried out and safe treatment protocols are adopted, always aiming at the quality of life and patient satisfaction.
\end{abstract}

Keywords: Osteoporosis; Diphosphonates; Osteonecrosis; Dentists. 


\begin{abstract}
Resumem
La osteoporosis es una enfermedad sistémica progresiva del esqueleto, caracterizada por la reducción de la densidad ósea y el deterioro de la microestructura del tejido óseo. Para el tratamiento, se ha recomendado el uso de bisfosfonatos (BF) como la terapia antirresortiva de primera elección. El objetivo de este trabajo es describir, a través de un caso clínico, un protocolo de atención odontológica para un paciente con Osteoporosis y utilizando BFF. El trabajo fue presentado y aprobado por el Comité de Ética en Investigación (CEP), opinión No: 3.646.508 / 2019. Paciente mujer de 30 años, negra, con osteoporosis, remitida para tratamiento odontológico. En el momento de la anamnesis, informó haber usado alendronato sódico $70 \mathrm{mg} /$ día. Después del examen clínico, las pruebas de laboratorio para la dosificación de CTX-I y la evaluación radiográfica, se presentó el plan de tratamiento sugerido: cirugía clínica de aumento de corona y posterior tratamiento endodóntico y rehabilitación oral del diente 25, que fue aprobado de inmediato por el paciente y firmado el Término de consentimiento informado (TCLE). Una semana antes del procedimiento quirúrgico de aumento clínico de corona, se solicitó la suspensión de alendronato sódico. Tras el tratamiento de endodoncia, se realizó la rehabilitación protésica, restableciendo la función masticatoria y estética de los dientes, devolviendo la autoestima del paciente. Se concluyó en el presente estudio que es posible realizar el tratamiento odontológico de los pacientes en terapia con bisfosfonatos de manera segura, siempre que se realice una anamnesis bien realizada y detallada y se adopten protocolos de tratamiento seguros, siempre con el objetivo de la calidad de vida y satisfacción del paciente.
\end{abstract}

Palabras clave: Osteoporosis; Bifosfonatos; Osteonecrosis; Odontologos.

\title{
1. Introdução
}

A osteoporose é uma doença sistêmica progressiva do esqueleto, caracterizada pela redução da densidade óssea e deterioração da microestrutura do tecido ósseo. Pesquisas de cunho epidemiológico apontam uma grande perda óssea após a quarta e quintas décadas de vida, em ambos os sexos, com intenso risco para mulheres em período pós-menopausa Assim, entendendo que a prevalência de osteoporose parece tem uma alteração com a idade do paciente, vários protocolos de tratamento vem sendo preconizados, sendo um destes o Alendronato de Sódio (Migliorati et al., 2005a).

Em pacientes com osteoporose é esperado que os Bifosfonatos (BFs), consigam conter a perda de estrutura óssea, além de realizar um aumento na densidade dos ossos, reduzindo o risco de fratura patológica, ocasionado pela progressiva perda de massa óssea. Estes medicamentos alteram o mecanismo de reabsorção e remodelação óssea e por este motivo, tem ação terapêutica nas seguintes patologias: pacientes com moderada a severa hipercalcemia associada com câncer; pacientes com lesões osteolíticas associadas ao câncer de mama e mieloma múltiplo em conjunto com quimioterapia antineoplásica e para lesões osteolíticas originárias de qualquer tumor sólido (Hilner et al., 2000; Nicolatou-Galitis et al., 2019).

Com o aumento dos casos de prescrição e utilização dos BFs, surgiram os primeiros relatos de complicações associadas à sua utilização, dos quais os mais comuns são em relação à mialgia e à esofagite e complicação que afeta exclusivamente a mandíbula e a maxila denominada de osteonecrose dos maxilares (Migliorati et al., 2005b; Bell et al., 2008; Granate-Marques et al., 2019)

A osteonecrose dos maxilares associada ao uso de medicamentos, é uma condição clínica não muito comum, e que está associada a prescrição e utilização pelos pacientes de BFs, e Denosumab. O principal sinal clínico desta condição é a presença de exposição óssea na mandíbula ou maxila. O fator etiologico é multifatorial e envolve a interação entre a exposição sistemica aos medicamentos antirreabsortivos, fatores sistêmicos (Fumantes, Idade Avançada, Anemia, Terapia prolongada com corticosteróides, Diabetes Mellitus) e ao trauma ou infeccção local na cavidade oral (Santos et al., 2016; Granate-Marques et al., 2019; Mendes et al., 2019).

Sobre os fatores de risco locais, são descritos na literatura como fatores predisponentes: exodontias, implante dental e cirurgia periapical. As exodontias representam um fator predisponente para a Osteonecrose em $69 \%$ a $86 \%$ dos casos (AlRahabi \& Ghabbani, 2018).

Outros fatores locais que representam fator de risco para a Osteonecrose associado aos medicamentos antireabsortivos, podem ser citados: A mandíbula, que representa duas vezes possibilidade de ser afetada que a maxila, devido a maior densidade mineral óssea. Outro fator seria a presença de condições inflamatórias como abscessos periodontais e 
dentoalveolares, que representam um risco 7 vezes maior. Pacientes que fazem uso de próteses removíveis estão expostos duas vezes mais quando comparados com pacientes que não utilizam BPs (Vilela-Carvalho et al., 2018).

Os pacientes em uso de BFs que apresentem sintomas não agrupados de lesões de osteonecrose, devem ser submetidos ao tratamento odontológico conservador. Complementarmente, devem ser medicados com fármacos sistêmicos quando necessário, incluindo tratamento analgésico para a dor e antibioticoterapia para o controle de infecções (Ruggiero et al., 2009; Vilela-Carvalho et al., 2018).

O papel do cirurgião-dentista nestes casos é essencial e deve fazer parte da primeira consulta: anamnese criteriosa, exame clínico extra e intraoral minucioso. Exames radiográficos irão colaborar no diagnóstico de cárie e de doença periodontal, na avaliação de terceiros molares impactados e no reconhecimento de metástases ósseas ou qualquer outro tipo de doença oral (Granate-Marques et al., 2019).

Além destes cuidados, um importante biomarcador ósseo que vem sendo utilizado para avaliar o risco da osteonecrose que é o CTX I (Interligadores C-Terminais do Colágeno tipo I). Trata-se de um marcador sérico de reabsorção óssea. Os resultados dizem respeito ao metabolismo ósseo do paciente descrito em picograma por mililitro. Os resultados são apresentados da seguinte forma: $100 \mathrm{pg} / \mathrm{ml}=$ alto risco, $150 \mathrm{pg} / \mathrm{ml}=$ risco moderado, $299 \mathrm{pg} / \mathrm{ml}=$ risco mínimo, $300 \mathrm{pg} / \mathrm{ml}=$ sem risco algum. Este exame é de fundamental importância pois auxilia na decisão do tratamento odontológico a ser empregado, quando o paciente é usuário de BFs, na tentativa de reduzir as chances da instalação da osteonecrose. Porém, mesmo com devidos cuidados a osteonecrose pode surgir (Marx et al., 2007; Silva, 2016).

Sendo assim, este trabalho se propõe a relatar caso clínico de paciente portadora de Osteoporose, em uso de BFs, e que procurou a clínica Odontologica de uma Universidade em Manaus/AM para realização de tratamento odontológico.

\section{Metodologia}

Trata-se de um relato de experiência descritivo e qualitativo (Pereira et al., 1995; Estrela, 2018) e revisão da literatura sobre Osteoporose e Uso de BFs. A paciente foi orientada sobre o relato de caso e logo em seguida, leu e assinou o TCLE

Este trabalho foi submetido e aprovado pelo Conselho de Ética e Pesquisa -CEP, com parecer nº 3.646.508/2019.

\section{Relato de Caso}

Paciente gênero feminino, 30 anos, melanoderma, portadora de Osteoporose diagnosticada a 3 anos, foi encaminhada para tratamento odontológico na Clínica Integrada de uma Universidade em Manaus/AM. Durante a anamnese, a paciente relatou fazer uso de Alendronato de Sódio70mg sendo um comprimido, uma vez por semana, pela manhã.

Ao exame clínico intraoral verificou-se que o elemento 25, apresentava extensa destruição coronária, com perda das paredes mesial e palatina (Figura 1). Não foi observada presença de mobilidade. Durante o exame clínico, constatou-se que o dente em questão não apresentou sensibilidade à palpação ou percussão. Os testes de sensibilidade pulpar ajudaram a definir o diagnóstico de Pulpite Irreversível, havendo indicação de tratamento endodôntico radical. 
Figura 1 - Elemento $25-1^{\text {a }}$ Consulta.

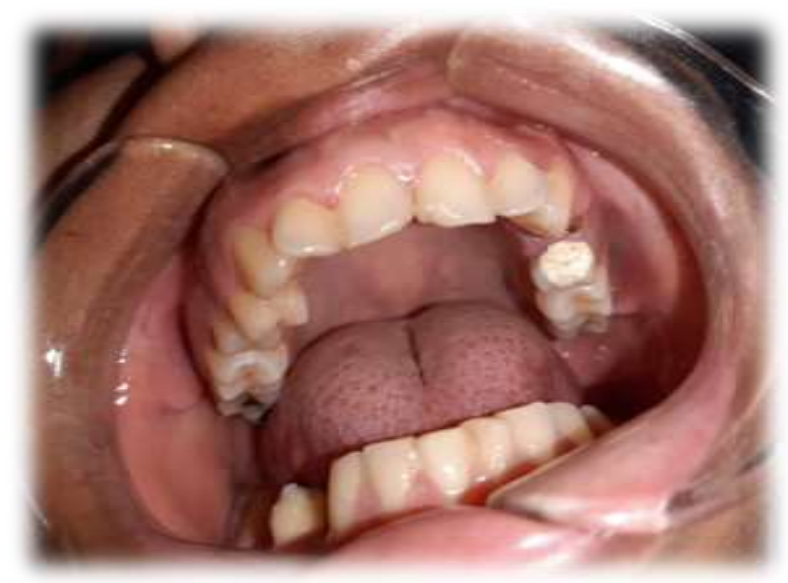

Fonte: Autores (2021).

Após a análise clínica e avaliação das radiografias Periapical (Figura 2) e panorâmica (Figura 3), observou-se na Radiografia Periapical que havia grande destruição coronária com comprometimento pulpar, mas sem lesão periapical. A radiografia panorâmica não apresentou alterações ósseas que indicassem áreas de reabsorção ou sequestro ósseo. Foi apresentado então o plano de tratamento sugerido: cirurgia de aumento de coroa clínica e posterior tratamento endodôntico, que foi prontamente aceito e autorizado pela paciente.

Figura 2 - Radiografia periapical elemento 25

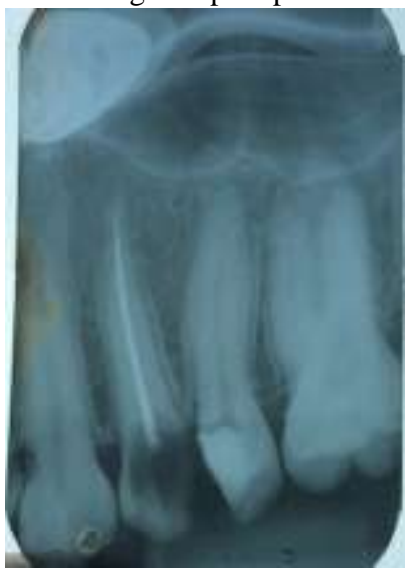

Fonte: Autores (2021).
Figura 3 - Radiografia panorâmica

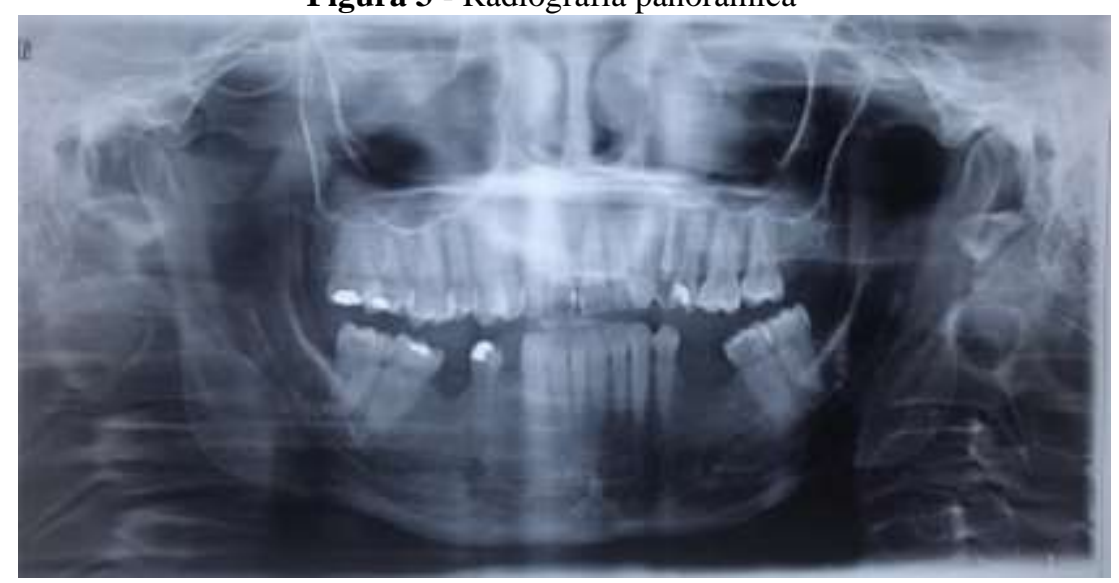

Fonte: Autores (2021).

Como exames pré-operatórios e complementares, foi solicitado à paciente que realizasse o exame laboratorial para o marcador CTX-I e Cálcio.

De acordo com os valores de referência, o valor de CTX-I para Mulher pré-menopausa é: 0,025-0,0573 ng/mL. O resultado apresentado pelo exame laboratorial da paciente foi de $\mathbf{0 , 3 4 5} \mathbf{n g} / \mathbf{m L}$, apresentando-se dentro dos padrões de normalidade e sem risco nenhum para Osteocrenose. Este resultado favoreceu a realização do procedimento cirúrgico, em conjunto com o exame de dosagem de Cálcio que também se apresentou dentro dos padrões de normalidade, considerando-se o valor de referência.

Com a apresentação de resultados satisfatórios dos exames laboratoriais, procedeu-se ao agendamento e realização da cirurgia de aumento de coroa clínica. A paciente foi encaminhada ao médico responsável por seu tratamento clínico, para avaliação e autorização para suspensão do medicamento antes do procedimento cirúrgico. Uma semana antes da realização do 
aumento de coroa clínica, foi solicitado à paciente a suspensão do Alendronato de sódio $70 \mathrm{mg}$, conforme literatura e protocolos da Associação Americana de Cirurgiões Orais e Maxilofaciais - AAOMS, que também preconizam a suspensão pós-cirúrgica, por quinze dias, até que inicie a cicatrização (Ruggiero et al., 2009).

Após o procedimento cirúrgico (Figura 4) foi realizada a prescrição medicamentosa, constituída de Amoxicilina $875 \mathrm{mg}$ + Clavulanato $125 \mathrm{mg}$, a cada 12 horas por 7 dias e Flancox $400 \mathrm{mg}$ a cada 8 horas por 3 dias. Prescreveu-se bochecho com Digluconato de Clorexidina a 0,12\% por 7 dias, 2vezes ao dia, pela manhã e antes de dormir.

Figura 4 - Aumento de Coroa Clínica.

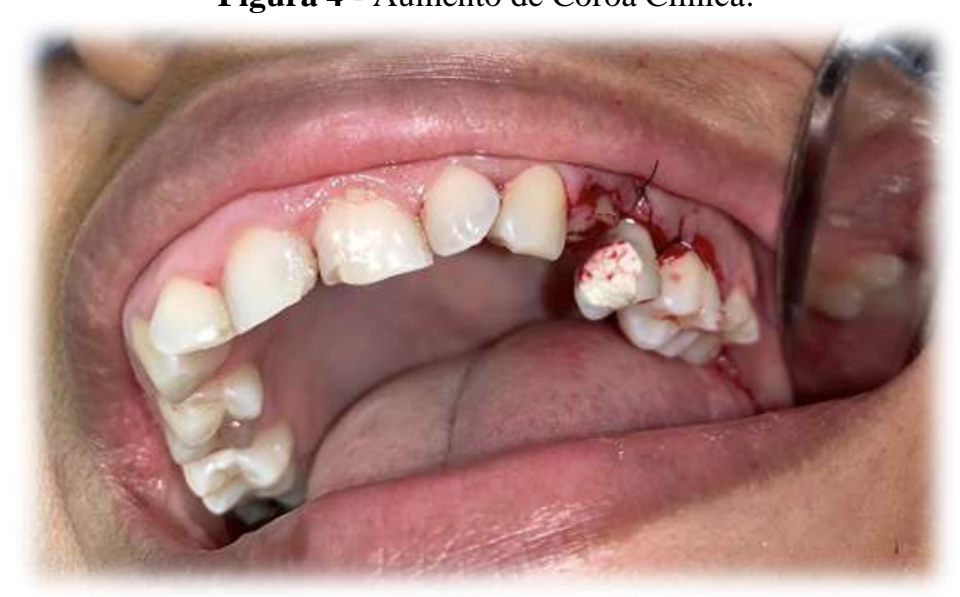

Fonte: Autores (2021).

Para o tratamento endodôntico foi realizada a técnica de instrumentação proposta pelo fabricante do Sistema ProTaper® (Dentsply (Maillefer, Ballaigues, Suíça) em uma única sessão e a obturação dos sistemas de canais radiculares foi feita com cimento obturador AH Plus (AH Plus, Dentsply/De Trey, GmbH Konstanz, Alemanha).

Após o tratamento endodôntico, foi feita a reabilitação protética com coroa provisória, restituindo a função mastigatória e estética dos dentes, devolvendo a autoestima à paciente e consequente melhora de sua qualidade de vida e autoconfiança (Figura 5).

Figura 5 - Reabilitação protética.

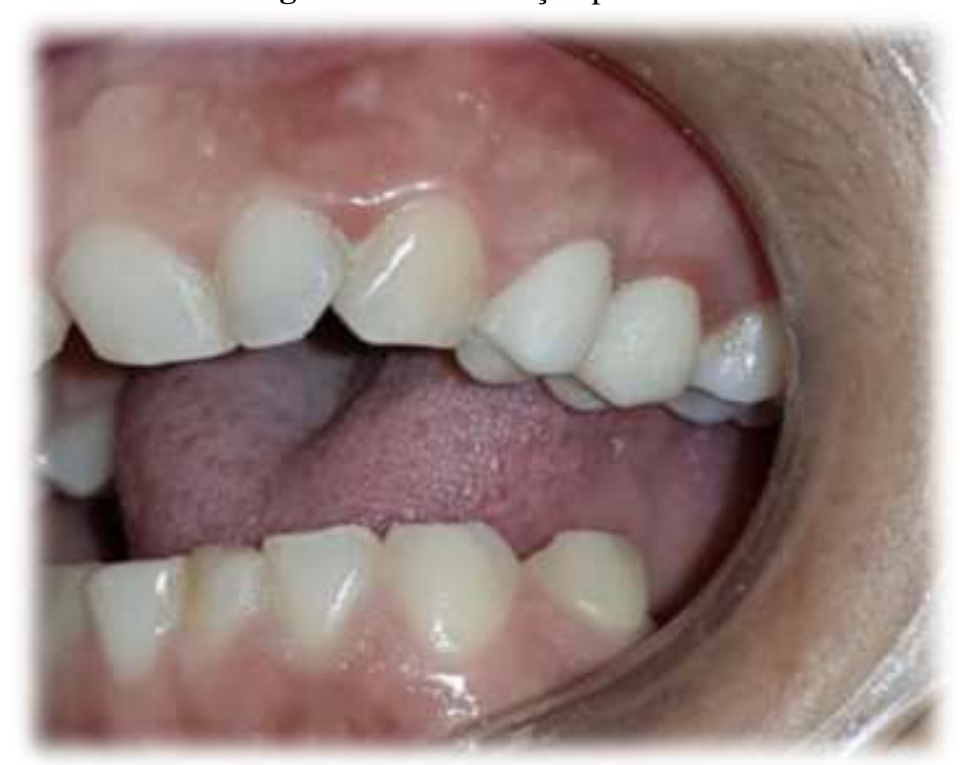

Fonte: Autores (2021). 


\section{Discussão}

A osteosporose é uma doença que se caracteriza pela diminuição da densidade óssea, induzindo o desenvolvimento de ossos vazios, delgados e frágeis, o que os torna mais propensos a fraturas. Esta condição está relacionada com um distúrbio na função dos osteoclastos, sobretudo em alterações na remodelação óssea. Ocorre então um aumento do número de células osteoclásticas, permitindo a reabsorção óssea, já que o volume de osteoblastos presentes realizando a neoformação óssea não superam a resposta dos osteoclastos. A consequencia são ossos fragéis e estrutura e formação de matriz ossea desorganizada (Ribeiro et al., 2018; Cremers et al., 2019).

Muitas terapias estão disponiveis para o controle da reaborção óssea em pacientes com osteoporose pós-menopausa, Doença de Paget, Câncer com metastase óssea e Mieloma múliplo. Os medicamentos anti-reabortivos como os bisfosfonatos, representam uma das muitas terapias propostas. (Zanata et al., 2014; Osborne et al., 2020).

O resultado esperado pela ação dos bifosfonatos em pacientes portadores de osteoporose é a contenção da perda de estrutura óssea, além do aumento da densidade, reduzindo o risco de fraturas resultantes da perda contínua de massa óssea. Estes medicamentos são depositados no osso e permanecem no interior da massa óssea por até 10 anos (Marx et al., 2007; Cólete et al., 2019).

Sabe-se no entanto que apesar de ser o medicamneto de $1^{a}$ escolha para o tratamento da Osteoporose, o uso prolongado dos BFs, pode levar a diversos efeitos colaterais, sendo a Osteonecrose o que traz mais prejuizo ao paciente, já que esta condição clinica além de corresponde a uma "área de exposição de osso necrótico que afeta mandíbula e maxila e que persiste por mais de 8 semanas em pacientes que estão tomando ou tomaram este medicamento" (Ruggiero et al., 2009). Os fatores de risco e consequente piora do quadro de Osteonecrose são: terapia com corticosteróides, Diabetes, Tabagismo e Etilismo (Medeiros et al., 2020).

Embora existam recomendações para interromper o uso dos Bifosfonato dois a três meses antes de procedimentos invasivos, a literatura é controversa sobre este assunto já que a suspensão não consiste em uma estratégia de redução de risco para a Osteonecrose, visto que esta medicamenção possui ação residual na medida em que se liga ao osso, permanecendo no tecido ósseo por anos. Para minimizar este problema, a literatura recomenda que sejam realizadas consultas odontológicas preventivas antes da terapia com os bisfosfonatos. O paciente poderá ser submetido à terapia periodontal para que alcance níveis de saúde satisfatórios, remoções cirúrgicas dentárias, adequação do meio bucal, bem como a adaptação satisfatória de próteses dentárias durante todo o percurso do tratamento por meio de bisfosfonatos, evitando, assim, a osteonecrose (VilelaCarvalho et al., 2018; Paiva et al., 2021). No presente estudo, a paciente já fazia uso de medicação anti-reabsortiva como protocolo para tratamento da Osteoporose. Foi instituido então anamnese detalhada e protocolo de tratamento seguro.

Os autores AlRahabi e Ghabbani, A (2018), pontuam que Quanto ao tratamento endodontico em pacientes em uso de BFs, é preferivel realiza-lo sempre que possivel, como alternativa á exodontia do elemento dental. Os autores concordam que a eliminação de microrganismos através dos procedimentos de limpeza e modelagem do sistema de canais radicualres tem como objetivo a cicatrização dos tecidos periapicais.

Um estudo onde foram avaliados a cicatrização da lesão periapical em pacientes em uso de Bifosfonatos, demonstrou que não houve diferenças estatisticamente significantes quando comparados com os pacientes do grupo controle e sem utilização da medicação em questão. $73,5 \%$ dos pacientes que faziam uso de BFs e foram submetidos ao tratamento endodontico radical, apresentaram diminuição da lesão periapical quando comparados com $81,6 \%$ dos pacientes que não faziam uso da medicação em questão (Hsiao, Glickman \& He., 2009). No presente estudo, houve a necessidade de Tratamento endodontico, mas não havia a presença de lesão periapical. A paciente foi orientada a fazer controle radiográfico de 6/6 meses por 2 anos, para acompanhamento do caso. 
CTX - Interligadores C-Terminais de colágenotipo I, é um marcador de remodelação óssea usado para medir o nível da atividade metabólica do tecido ósseo. Vem sendo solicitado como exames complementares em pacientes que tem a indicação de procedimentos eletivos invasivos e que fazem uso de anti-reabsortivos, ajudando portanto no diagnóstico e risco de desenvolvimento da Osteonecrose dos Maxilares. Os valores considerados normais são de 300-600 pg/ml, e valores menores que $150 \mathrm{pg} / \mathrm{ml}$ indicam alto risco para o desenvolvimento de Osteonecrose. Este exame foi solicitado previamente ao procedimento cirurgico, pela paciente relatada no caso clinico objeto deste estudo (Silva, 2016; Pinto-Junior et al., 2017).

Diante do exposto, fica claro que um dos fatores etiológicos responsável pelo desencadeamento da Osteonecrose, são os procedimentos cirúrgicos odontológicos, visto que ocorre exposição do tecido ósseo necrótico ao meio bucal. No entanto, existe a possibilidade de prevenção da ocorrência desta condição, induzida por bisfosfonatos segundo vários autores, havendo a possibilidade inclusive de tratamento de pacientes que usam esta medicação e necessitam de uma intervenção odontológica invasiva. Alem disto, o consenso entre o cirurgiãodentista, o paciente e o seu médico que o assiste, é extremamente importante antes de se iniciar o tratamento odontológico. No presente trabalho o médico assistente foi consultado e inclusive suspendeu a medicação anti-reabsortiva para a realização do procedimento cirurgico (Santos et al., 2016; Mendes et al., 2019; NicolatouGalitis et al., 2019).

\section{Considerações Finais}

O cirurgião dentista tem a responsabilidade de propor aos seus pacientes usuários de Bisfosfonato, tratamentos preventivos que não envolvam procedimentos cirúrgicos. Caso haja necessidade de tratamento invasivo, este deve ser realizado em comum acordo com o médico que acompanha o paciente, além de realização de anamnese cuidadosa e detalhada. Um bom planejamento nestes casos, aliado ao conhecimento técnico-científico é capaz de reabilitar o paciente e permitir que o mesmo tenha com qualidade de vida. Fica o alerta para a classe odontológica sobre a importância do trabalho em equipe com outros profissionais com o intuito de promover um diagnóstico precoce dos casos de Osteonecrose dos Maxilares e o conhecimento sobre os protocolos preventivos a esta condição que deve ser discutido e analisado pela equipe responsável pelo paciente.

\section{Referencias}

AlRahabi, M. K., \& Ghabbani, H. M. (2018). Saudi Medical Journal, 39 (3): 232-238.

Bell, B. M., \& Bell, R. E. (2008). Bifosfonatos orais e implantes dentários: um estudo retrospectivo. Journal Of Oral And Maxillo Facial Surgery, 5(66): 4-10.

Coléte, J.Z., Hadad, H., Momesso, G. A. C., Santos Filho, H., Breno dos Reis, F., \& Garcia Júnior, I. R. (2019). Implantes em pacientes com osteonecrose dos maxilares associado ao uso de bifosfonatos: relato de caso e revisão de literatura. Archives of Health Investigation, 8(1):20-27.

Cremers, S., Drake, M. T., Ebetino, F. H., Bilezikian, J. P., \& Russell, R. (2019). Pharmacology of isphosphonates. British journal of clinical pharmacology, 85(6):1052-1062.

Estrela, C. Metodologia científica: ciência, ensino, pesquisa. (3a ed.), Artes Médicas, 2018.

Granate-Marques, A., Polis-Yanes, C., Seminario-Amez, M., Jané-Salas, E., \& López-López, J. (2019). Medication-related osteonecrosis of the jaw associated with implant and regenerative treatments: Systematic review. Medicina Oral, Patologia Oral, Cirugia Bucal, $24(2):$ e195-e203.

Hillner, B. E., et al (2000). American Society of Clinical Oncology guideline on the role of bisphosphonates in breast cancer. American Society of Clinical Oncology Bisphosphonates Expert Panel. Journal of Clinical Oncology, 18(6):1378-91.

Hsiao, A., Glickman, G., \& He, J. (2009). A retrospective clinical and radiographic study on healing of periradicular lesions in patients taking oral bisphosphonates. Journal of Endodontics, 35(11):1525-8.

Marx, R. E., Cillo, J. E., \& Ulloa, J. J. (2007). Oral Bisphosphonate-Induced Osteonecrosis: Risk Factors, Prediction of Risk Using Serum CTX Testing, Prevention, and Treatment. Journal of oral and Maxillofacial Surg, 65(12):2397-2410.

Medeiros, I. L., Rebouças, S. C. R., Souza Junior, F. A., Araújo Neto, G. H., Pinheiro, S. S., \& Barroso, M. L. F. (2020). Implantes dentários e a osteonecrose dos maxilares associado ao bifosfonato: uma revisão integrativa. Research, Society and Development, 9(10): e6519108622.

Mendes, V., et al (2019). Impact of bisphosphonate therapy on dental implant outcomes: An overview of systematic review evidence. International Journal of Oral and Maxillofacial Surgery, 48(3):373-381. 
Research, Society and Development, v. 10, n. 9, e22810918103, 2021

(CC BY 4.0) | ISSN 2525-3409 | DOI: http://dx.doi.org/10.33448/rsd-v10i9.18103

Migliorati, C. A., et al (2005a). Bisphosphonate-associated osteonecrosis of mandibular and maxillary bone: an emerging oral complication of supportive cancer therapy. Cancer; 104(1):83-93.

Migliorati, C. A., et al (2005b). Managing the care of patients with bisphosphonate-associated osteonecrosis: an American Academy of Oral Medicine position paper. Journal of American Dental Association, 136(12):1658-68.

Nicolatou-Galitis, O., Schiødt, M., Mendes, R. A., Ripamonti, C., Hope, S., Drudge-Coates, L., Niepel, D., \& Van den Wyngaert, T. (2019). Medicationrelated osteonecrosis of the jaw: definition and best practice for prevention, diagnosis, and treatment. Oral Surgery Oral Medical Oral Pathology Oral Radiology, 127(2):117-135.

Osborne, P. R., Mendes, B., Faverani, L. P., Alexandre, C. N., \& Jesus, G. P. (2020). Osteonecrose Maxilar Induzida por medicamento anti-reabsortivo (Bisfosfonato) em Paciente Portador de Mieloma Múltiplo - Relato de caso. Research, Society and Development, 9(11): e1179119415.

Paiva, C. L.O. C., Silva, N. M. M., Oliveira, T. A., Souza, J. A. N., Pereira, G. M.A., \& Souza Júnior, F.A. (2021). Osteonecrose dos maxilares relacionada ao uso de medicamentos: Revisão sistemática. Research, Society and Development, 10(5): e15510514740.

Pereira, M. G. Epidemiologia: teoria e prática. Guanabara Koogan, 1995.

Pinto Junior, A. A. C., et al (2017). Osteonecrose dos Maxilares Associada ao uso de Bisfosfonatos. Rev. Cir. Traumatol. Buco-Maxilo-Fac., $17(1)$ : 40-45.

Ribeiro, G. H., et al (2018). Osteonecrose da mandíbula: revisão e atualização em etiologia e tratamento. Brazilian Journal of Otorhinolaryngology. 84 (1) $102-108$.

Ruggiero, S. L., et al. Task Force on American Association of Oral and Maxillofacial Surgeons position paper on bisphosphonate-related osteonecrosis of the jaw - 2009 update. Aust Endod J. 2009;35(3):119-30.

Santos, L. C. S., et al (2016). Influência Do Uso De Bisfosfonatos em Pacientes Submetidos a Implantes Dentários - Revisão da Literatura. Revista Bahiana de Odontologia, 7(1):22-30.

Silva, S. E. (2016). Uso do exame laboratorial CTX na avaliação de prognóstico cirúrgico odontológico em pacientes com histórico de uso de bisfosfonato. Porto velho: Universidade São Lucas.

Song, M. (2019). Dental care for patients taking antiresorptive drugs. A literature review. Restorative Dentistry and Endodontics, 44(4):e42-58.

Vilela-Carvalho, L. N., et al (2018). Osteonecrose dos maxilares relacionada ao uso de medicações: Diagnóstico, tratamento e prevenção. CES Odontologia, $31(2): 48-63$

Zanata, A., Felin, G. C., De Bona, M. C., Sawazaki, R., \& De Conto, F. (2014). Osteonecrose mandibular associada ao uso de bisfosfonato de sódio em paciente com mieloma múltiplo. Revista Portuguesa de Estomatologia e Medicina Dentaria e Cirurgi Maxilofacial, 55(2):115-20. 